\title{
Einwirkung von schwefliger Säure auf Tiglinaldehyd
}

\author{
von \\ Felix Hugo Hayman. \\ Aus dem chemischen Laboratorium des Prof. Ad. Lieben \\ an der k. k. Universität in Wien.
}

(Vorgelegt in der Sitzung am 25. October 1888.)

In einer kürzlich erschienenen Arbeit von E. Ludwi g ${ }^{1}$ wurde die Einwirkung von schwefliger Säure auf Metbyläthylacrolein dargelegt. Der Verfasser bediente sich bei seinen. Versuchen der Methoden, welche Zeisel und Alic bei einer ähnlichen Arbeit über den Crotonaldehyd (welche bis jetzt nicht publicirt ist) für vortheilhaft befunden. Einer Anregung. des Herrn Professors Lie ben folgend, untersuchte ich das Verbalten des von Lieben und $\mathrm{Z}$ eisel durch Condensation von Acet- und Propionaldehyd erhaltenen Tiglinaldehydes ${ }^{2}$ gegen schweflige Säure. Ich fand dabei, dass man durch Behandlung mit schwefliger Säure, je nach den Versuchsbedingungen einen sulfonirten Valeraldehyd oder eine Oxypentandisulfonsäure erhält, von denen die letztere durch Abspaltung von schwefliger Säure in den ersteren überzugehen vermag.

Der sulfonirte Valeraldehyd wird durch Oxydation in sulfonirte Valeriansäure, durch nascirenden Wasserstoff aber in sulfonirten Amylalkohol umgewandelt.

Als Ausgangsmaterial diente mir ein Tiglinaldehyd, der nach der Methode von Lieben und Zeisel im hiesigen Laboratorium dargestellt worden war.

1 Monatsh. f. Chemie, 1888, S. 658 ff.

2 Monatsh. f. Chemie 1886, S. 53 ff. 


\section{Einwirkung wässeriger schwefliger Säure auf Tiglinaldehyd bei gewöhnlicher und bei höherer Temperatur.}

Mit Berïcksichtigung der Beobachtungen von Zeisel und Alic durfte von vornherein angenommen werden, dass auch der Tiglinaldehyd schweflige Säure in zweifacher Weise zu addiren vermag. Einerseits konnten $\mathrm{H}$ und $\mathrm{SO}_{3} \mathrm{H}$ an je eines der doppelt gebundenen Kohlenstoffatome treten, anderseits an das Sauerstoffund Kohlenstoffatom der Formylgruppe, diese in den Atomeomplex $\mathrm{CH} . \mathrm{OH} . \mathrm{SO}_{3} \mathrm{H}$ umwandelnd.

Der Hauptzweck der nachfolgenden Versuche lag nun darin, zu beobachten, unter welchen Umständen der eine und der andere Fall eintritt. Zwei parallele Darstellungswege der Aldehydsulfonsäuren wurden zu diesem Ende eingeschlagen. Zuerst wurden $5 \mathrm{~g}$ Tiglinaldehyd mit circa $50 \mathrm{~cm}^{3}$ Wasser unter Eiskühlung in einer Flasche mit schwefliger Säure übersättigt. Das Gefäss wurde fest verstopft, geschiittelt und bei Zimmertemperatur stehen gelassen. Nach drei Stunden war vollständige Lösung eingetreten. Die Flüssigkeit wurde (unter Eisküllung) mit Baryumcarbonat neutralisirt und filtrirt. Das anfangs klare Filtrat schied schon beim Stehen, mehr noch bei dem darauffolgenden Einengen im Vacuum, wobei eine Temperatur von $35^{\circ}$ nicht überschritten wurde, eine beträchtliche Menge von Baryumsulfit aus, welches nur durch Zersetzung des. Baryumsalzes einer wenig beständigen Sulfonsäure entstanden sein konnte.

Das Filtrat vom Baryumsulfit hinterliess beim Eindampfen im Vacuum einen gummiartigen Rückstand von einer Zusammensetzung, Gie für ein Gemenge von oxypentandisulfonsaurem Baryum und valeraldehyd-sulfonsaurem Baryum stimmte. Man kann also behaupten, dass durch die Einwirkung von schwefliger Säure bei niedriger Temperatur entweder ein Gemenge von Disulfonsäure und Monosulfonsäure entstanden ist, oder vielleicht nur Disulfonsäure, welche nach der Neutralisation theilweise im Sinne folgender Gleichung in das Baryumsalz des sulfonirten Valeraldehydes, Baryumsulfit und schweflige Säure zerfällt:

$$
2 \mathrm{C}_{5} \mathrm{H}_{10} \mathrm{O}\left(\mathrm{SO}_{3}\right)_{2} \mathrm{Ba}=\left(\mathrm{C}_{5} \mathrm{H}_{9} \mathrm{OSO}_{3}\right)_{2} \mathrm{Ba}+\mathrm{BaSO}_{3}+\mathrm{H}_{2} \mathrm{SO}_{3}
$$


$0.47125 \mathrm{~g}$ der vacuumtrockenen Substanz gaben $0.376 \mathrm{~g} \mathrm{CO}_{2}$ und $0 \cdot 15325 g \mathrm{H}_{2} \mathrm{O}^{1}$

$0 \cdot 3325 g$ der vacuumtrockenen Substanz gaben $0 \cdot 1784 g \mathrm{BaSO}_{4}$. Berechnet für

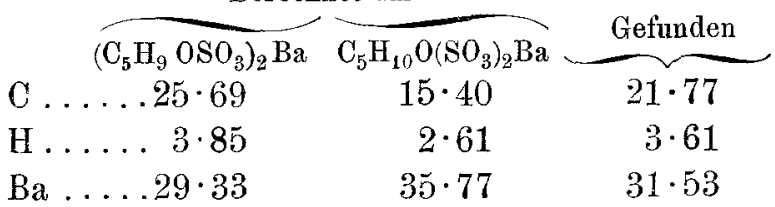

Um zu erfahren, ob durch Einwirkung von schwefliger Säure bei höherer Temperatur vielleicht ausschliesslich Disulfonsäure gebildet wird, wurden bei einem anderen Versuch $10 \mathrm{~g}$ des Aldehyds unter Eiskiühlung mit schwefliger Säure übersättigt und hierauf im zugeschmolzenen Rohre circa vier Stunden auf $65^{\circ}$ erhitzt. Es war vollständige Lösung eingetreten, der Rohrinhalt sah bräunlich aus. Die weitere Behandlung war dieselbe wie friiher.

Auch diesmal schied sich während des Eindestillirens und nachfolgendenAbdampfens Baryumsulfitin bedeutender Menge ans. Es musste daher der Versuch, ein Salz der Disulfonsäure zu isoliren, wegen der grossen Zersetzlichkeit dieser Verbindungen aufgegeben werden.

Hingegen liess sich das Baryumsalz einer Sulfonsäure von der Zusammensetzung $\mathrm{C}_{5} \mathrm{H}_{9} \mathrm{OSO}_{3} \mathrm{H}$ leicht isoliren, indem ein solches Gemenge von mono- und disulfonsaurem Baryum, wie es bei den bereits beschriebenen Versuchen erhalten wird, bei Gegenwart von iberschiissigem Baryumcarbonat anhaltend gekocht wurde. Dies geschah in einer Kohlensäureatmosphäre, um eine Oxydation des erwarteten sulfonirten Aldelyds hintanzuhalten. Das gebildete Baryumsulfit, sowie das iubersehüssige Baryumearbonat wurden abfiltrirt; das vollkommen neutrale Filtrat wurde imVacuum über Schwefelsäure eingedunstet, der Rückstand bei $100^{\circ}$ bis zur Gewichtsconstanz getrocket. Das so erhaltene Salz zeigte indess jetzt beim Auflösen einer Probe in Wasser deutlich saure Reaction. Wie später gezeigt werden wird, lag hier das Baryumsalz eines sulfonirten Valeraldehyds vor, welches

1 Diese und alle nachfolgenden Verbrennungsanalysen wurden mit Bleichromat ausgeführt. 
leicht durch Oxydation in das Salz der entsprechenden sulfonirten Valeriansäure überzugehen vermag. Diese Eigenschaft erklärt auch das Sauerwerden des ursprünglich neutralen Salzes.

$0 \cdot 1715 g$ Substanz gaben $0 \cdot 15375 g \mathrm{CO}_{2}$ und $0.0625 g \mathrm{H}_{2} \mathrm{O}$. $0 \cdot 359 g$ Substanz gaben $0 \cdot 17275 g \mathrm{BaSO}_{4}$.

Berechnet für

$\begin{array}{lr}\stackrel{\left.\mathrm{C}_{5} \mathrm{H}_{9} \mathrm{OSO}_{3}\right)_{2} \mathrm{Ba}+\mathrm{H}_{2} \mathrm{O}}{\mathrm{C} \ldots \ldots .24 \cdot 79} \quad \frac{\text { Gefunden }}{24 \cdot 45} \\ \mathrm{H} \ldots \ldots 4 \cdot 12 & 4 \cdot 05 \\ \mathrm{Ba} \ldots \ldots 28 \cdot 24 & 28 \cdot 29\end{array}$

Diese Analyse bedurfte einer Correctur. Wie schon erwähnt, hatte sich die Formylgruppe des Valeraldehyd-sulfonsauren Salzes durch freiwillige Oxydation zum Theil in die Carboxylgruppe verwandelt. Die dadurch neu entstandene Acidität des Salzes wurde durch Titriren mit Kalilauge ermittelt, und daraus die Menge des aufgenommenen Sauerstoffs berechnet. Dabei entsprach je einem Molekiil Kali ein Atom Sauerstoff. Zog ich die so gefundene Gewichtsmenge Sauerstoff von dem Gewichte der Substanz ab, so erhielt ich die eigentliche Gewichtsmenge, welche zur Analyse verwendet worden war. Mit dieser Correctur stellen sich die Ergebnisse der Elementaranalyse und der Baryumbestimmang wie folgt:

$0.17 \mathrm{~g}$ Substanz (das ist nach Abzug von $0.0015 \mathrm{~g}$ für aufgenommenen Sauerstoff) gaben $0 \cdot 15375 \mathrm{Co}_{2}$ und $0.0625 \mathrm{~g}$ $\mathrm{H}_{2} \mathrm{O}$.

$0.356 g$ Substanz (nach Abzug von $0.003 g$ für aufgenommenen Sauerstoff) gaben $0 \cdot 17275 g \mathrm{BaSO}_{4}$.

Berechnet für

\begin{tabular}{|c|c|}
\hline$\left(\mathrm{C}_{5} \mathrm{H}_{9} \mathrm{OSO}_{3}\right)_{2} \mathrm{Ba}+\mathrm{H}_{2} \mathrm{O}$ & Gefunden \\
\hline C. . .24.79 & $24 \cdot 67$ \\
\hline $\mathrm{H} \ldots \ldots 4 \cdot 12$ & $4 \cdot 09$ \\
\hline Ва....28.24 & $28=53$ \\
\hline
\end{tabular}

Zu meinem Bedauern fehlte mir die Zeit, durch sorgfältige Darstellung und Analyse eines neten Präparates diese Analysen 
$z u$ controliren, welche an einem in kleiner Menge erhaltenen Präparate ansgefuihrt worden waren.

Was die Disulfonsäure betrifft, so ist ihre Existenz an anderer Stelle nachgewiesen, nämlich bei der Oxydation mit Brom. Dabei scheidet sich Baryumsulfat aus, was nur unter der Voraussetzung cines disulfonsauren Salzes möglich ist. Auch dure die Thatsache, dass das Baryumsalz der präsumptiven Disulfonsäure beim Erhitzen Baryumsulfit ausscheidet, erscheint ihre Existenz sicher gestellt.

Es lässt sich nicht feststellen, ob bei Einwirkung von sehwefliger Säure auf Tiglinaldehyd bei höherer oder auch bei niederer Temperatur bloss die Disulfonsäure entsteht, welche nachträglich dissociirt, oder ein Gemenge von Di- und Monosulfonsäure. Es ist aber bewiesen dass das disulfonsaure Baryumsalz der Dissociation zu monosulfonsaurem Baryum, Baryumsulfit und schwefliger Säure bei gewöhnlicher Temperatur theilweise bei $100^{\circ}$ vollständig anheimfällt.

\section{Reduction der Tiglinaldehydsulfonsäuren.}

Eine Partie von $20 \mathrm{~g}$ Tiglinaldehyd wurde mit schwefliger Säure gesättigt und eine Nacht bei Zimmertemperatur stehen gelassen. Bei diesem Verfahren entsteht, wie früher gezeigt wurde, ein Gemisch von Monosulfonsäure und Disulfonsäure. In dieses Product wurde Natriumamalgam, und zwar mehr als die doppelte Menge, die darch die Theorie gefordert wird, eingetragen. Das Amalgam, welches circa vierprocentig war, wurde in kleinen, gewogenen Partien zugesetzt und durch Hinzufïgen der entsprechenden Mengen einer titrirten Schwefelsäure die Flüssigkeit immer schwach saner erhalten. Nach beendeter Reaction wurde das Quecksilber entfernt, mit Natronlauge neubralisirt und zur Hälfte abdestillirt. Dabei giengen äusserst geringe Mengen eines öligen, stark riechenden Productes iiber. Die Flussigkeit, weiter eingeengt und mit dem gleichen Volumen Alkohol versetzt, schied den grössten Theil des gebildeten Natriumsulfats ab. Die Mutterlange des erhaltenen Natriumsulfats wurde nach entsprechender Einengung der Behandlung mit Alkohol so oft unterworfen, bis die Schwefel- 
säurereaction im Filtrat der letzten Ausscheidung verschwunden war. Dabei erhielt ich eine gelbe, syrupöse Masse, welche das (wahrscheinlich nicht reine) Natriumsalz eines sulfonirten Amylalkohols darstellte.

Dass wirklich diese Verbindung vorlag, erhellt daraus, dass diese Substanz, mit Kalk erhitzt. neben schwefligsaurem und schwefelsaurem Calcium, wie ans Nachfolgendem zu ersehen ist, ein Gemenge von Tiglylalkohol und Amylalkohol lieferte.

Mit Ätzbaryt gekocht zeigte der Körper einen nicht sehr starken, aber doch deutlich erkennbaren Geruch nach Tiglinaldeliyd, was offenbar von der Reduction entgangener Substanz herriihrte.

Da unter diesen Umständen eine Analyse kaum zu scharf stimmenden Zahlen führen konnte, wurde davon abgesehen, und das Product sofort einer Behandlung im oberwähnten Sinne unterworfen.

$\mathrm{Zu}$ diesem Ende wurde der Syrup in frisch gebrannten Kalk aufsaugen gelassen, das Gemisch auf dem Wasserbad zur Trockene gebracht, hierauf mit überschïssigem frisch gebranntem Kalke verrieben und in mehreren Partien der trockenen Destillation aus Glasröhren unterworfen. Dies geschah mit Vortheil in einem Wasserstoffstrom. Das Destillat, ein braunes Öl, wurde durch Destillation mit Wasserdampf vorlänfig gereinigt, wobei geringe Mengen einer braunen, harzigen Substanz hinterblieben. Die Ausbente an Rohproduct betrug etwa 50 Procent des Aldehyds. Das Öl wurde mit Potasche getrocknet und fractionirt destillirt. Ein grosser Theil gieng zwischen $120-140^{\circ}$ farblos über. Der höher siedende Rückstand war nicht unbeträchtlich. Derselbe wurde nicht weiter berücksichtigt.

Lieben und Zeisel haben für das Gemenge von gesättigtem. Alkohol $\mathrm{C}_{5} \mathrm{H}_{12} \mathrm{O}$ und ungesättigtem Alkohol $\mathrm{C}_{5} \mathrm{H}_{10} \mathrm{O}$, das sie durch directe Reduction des T'iglinaldehyds mit Eisenfeile und Essigsäure erhielten, eine Siedetemperatur von $125-135^{\circ}$ gefunden.

In der Voraussetzung, dass wirklich ein solches Gemenge vorlag; wurde diese Fraction in der ungefähr fünfigfachen 
Menge Wassers gelöst, mit Brom bis zur eintretenden Gelbfärbung versetzt und hierauf am Rückflusskühler durch 12 Stunden gekocht. Durch diese Behandlung sollte etwa vorhandenerTiglylalkohol durch das Bromadditionsproduct hindurch in das zugehörige Pentenylglycerin umgewandelt werden, während etwa vorhandener Amylalkohol dabei keine Veränderung erfahren konnte. Die Flìssigkeit, welche sich gelblich gefärbt hatte, wurde zur Hälfte abdestillirt; dabei gieng ein gelbes $0 ̈ 1$ ỉber. Da Lieben und Zeisel die Beobachtung gemacht hatten, dass durch Einwirkung von Wasser auf die Dibromadditionsproducte der ungesättigten Alkohole immer auch eine kleine Menge der zugehörigen ungesättigten Aldehyde gebildet wird, wurde das Öl, welches durch eine Reihe von Destillationen aus dem wässerigen Antheil des ersten Destillates abgeschieden worden war, mit Natriumbisulfitlösung geschüttelt. Sein Volumen nahm bei dieser Behandlung äusserst wenig ab. Da wenig Aussicht vorhanden war, das Öl, dessen Menge durch die Präparation sehr abgenommen hatte, zur Analyse genügend trocken und rein $z \mathfrak{u}$ erhalten, wurde es durch Erhitzen mit Essigsäureanhydrid in das leichter zu reinigende Acetat über gefïhrt und dieses nach entsprechender Reinigung der Analyse unterworfen. Der vermuthliche Alkohol wurde mit der theoretischen Menge Essigsäureanhychid im zugeschmolzenen Rohre einige Stunden lang auf $140^{\circ}$ erhitzt. Der Rohrinhalt wurde mit Sodalösung, dann mit Wasser gewasehen, endlich mit Chlorcalcium getrocknet and destillirte zwischen $138-143^{\circ}$ ïber. (uncorrigirt). Lieben und Zeisel fanden für das Acetat des Methyläthylearbincarbinols den Siedepunkt 141 -6 (corrigirt). Die Elementaranalyse zeigte die erwarteten Zahlen.

I. $0 \cdot 15975 \mathrm{~g}$ Substanz gaben $0.378 \mathrm{~g} \mathrm{CO}_{2}$ und $0 \cdot 151 \mathrm{~g} \mathrm{H}_{2} \mathrm{O}$. II. $0 \cdot 165 g$ Substanz gaben $0 \cdot 388 g \mathrm{CO}_{2}$ und $0 \cdot 153 g \mathrm{H}_{2} \mathrm{O}$.
$\underbrace{\text { Berechet fü }}_{\mathrm{C}_{5} \mathrm{H}_{11} \mathrm{OC}_{2} \mathrm{H}_{3} \mathrm{O}}$
C....664.41
$\underbrace{\text { I. Gefunden }}_{64 \cdot 53} \frac{\text { II. }}{64 \cdot 13}$
$\mathrm{H} \ldots \ldots 10 \cdot 77$
$10 \cdot 50 \quad 10 \cdot 30$

Die Analyse allein konnte allerding's nicht entscheiden, $o b$ nicht der Verbindung vielleicht der Essigester eines ungesättigten 
Amylalkohols beigemengt sei. Dies ist pun nach den Erfahrungen von Lieben und Zeisel wenig wahrscheinlich; nichtsdestoweniger wurde die Abwesenheit irgend einer ungesättigten Verbindung noch dadurch erwiesen, dass ich mich ïberzeugte, dass einige Tropfen der Substanz schon durch das in einem Tropfen Bromwassers enthaltene Brom bleibend gelb gefärbt wurden und demnach nicht die Eigenschaft zeigten, Brom additionell aufzunehmen.

Wenn das Rohproduct der trockenen Destillation der reducirten sulfonsauren Natriumsalze mit Kalk neben dem soeben besprochenen gesättigten Alkohol noch einen ungesättigten enthalten hatte, musste dieser neben Bromwasserstoffsäure als Pentenylglycerin in jener Flüssigkeit zu finden sein, die nach dem Bromiren, Kochen am Rückflusskiuhler und Abdestilliren zur Hälfte als Rückstand erhalten worden war. Der Weg zur Auffindung des Glycerins war durch die Arbeiten von Lieben und Zeis el vorgeschrieben. Die Flüssigkeit wurde mit Bleioxyd in der Wärme digerirt, um den grössten Theil der Bromwasserstoffsäure zu binden. Hierauf wurde vom Bromblei und überschüssigen Bleioxyd abfiltrirt und das Filtrat mit Schwefelwasserstoff rom Blei befreit, der Schwefelwasserstoff verjagt, die letzten Reste von Bromwasserstoff durch Silberoxyd gefällt, geringe Mengen in Lösung gegangenen Silbers wieder mit Sehwefelwasserstoff entfernt, endlich dieser wieder verjagt und concentrirt. Die concentrirte Flüssigkeit wurde zur Reinigung mit Alkoholäther extrabirt. Die alkoholisch-ätherische Lösung hinterliess beim Abdunsten einen schwach gefärbten Syrup, der im Vacuum bis zur constanten Gewichtsabnahme getrocknet wurde. Derselbe zeigte süsslichen Geschmack und ergab bei der Analyse Zahlen, die auf die Formel $\mathrm{C}_{5} \mathrm{H}_{9}(\mathrm{OH})_{3}$ stimmten. $0 \cdot 11325 g$ Substanz gaben $0 \cdot 20725 g \mathrm{CO}_{2}$ und $0.0985 g \mathrm{H}_{2} \mathrm{O}$.

$$
\begin{aligned}
& \text { Berechnet für } \\
& \begin{array}{r}
\text { C....50.00 } \\
\mathrm{H} \ldots \ldots .10 \cdot 00
\end{array} \quad \frac{\mathrm{C}_{5} \mathrm{H}_{22} \mathrm{O}_{3}}{49 \cdot 90}
\end{aligned}
$$

Werfen wir einen Riuckblick auf die Reihe von Veränderungen, vermittelst welcher das ursprüngliche Gemisch von 
Oxypentandisulfonsäure und Valeraldehydsulfonsäure schliesslich in ein Gemenge von Amylalkohol nnd Tiglylalkohol übergefülurt wurde, so sehen wir nacheinander folgende Reihe von Reactionen sich abspielen:

I. Valeraldehydsulfonsäure wird durch Aufnahme von 2wei Atomen Wasserstoff in Oxypentansulfonsäure umgewandelt.<smiles>CCC(C)(C(=O)O)C(=O)O</smiles>

II. Oxypentandisulfonsäure wird durch Aufnahme ron Wasserstoff in Oxypentansulfonsäure und schweflige Säure gespalten.

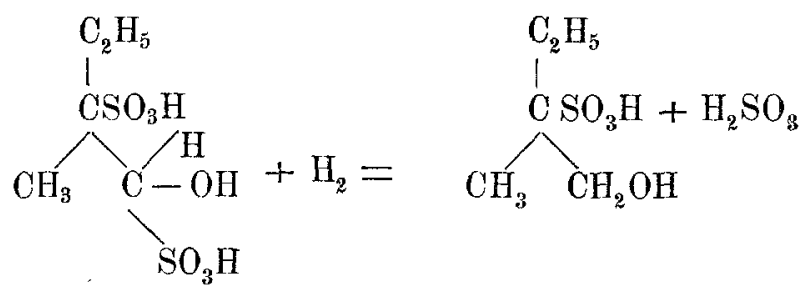

III. Durch Erhitzen mit $\mathrm{Ca}(\mathrm{OH})_{2}$ wird das oxypentansulfonsaure Natrium, indem die Elemente des sauren Natrinmsulfit abgespalten werden und die mit ihren verbunden gewesenen Kohlenstoffatome gegenseitig in doppelte Bindung treten, der Tiglylalkohol gebildet.<smiles>CC(CO)=C(C)COC(=O)C(C)CO</smiles> 
IV. Gleichzeitig mit dem sub III angedeuteten Processe spielt sich ein zweiter zur Bildung des Methyläthylcarbincarbinols führender $a b$ :

2

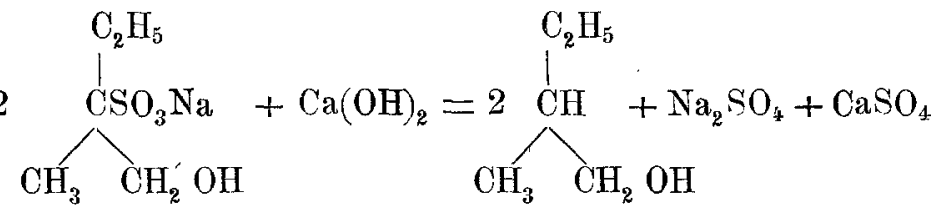

Der Tiglylalkohol wurde in Dibromid und Pentenylglycerin, der erhaltene Amylalkohol in Amylacetat übergeführt.

In vorstehenden Gleichungen wurde, ohne dass bis jetzt ein Beweis dafür erbracht worden wäre, angenommen, dass der eine Schwefelsäurerest an das tertiäre Kohlenstoffatom gebunden ist. Diese Annahme hat sehr viel Wahrscheinlichkeit für sich, wenn man bedenkt, dass auch Halogenwasserstoffsäuren, Schwefelsäure und ähnliche Verbindungen, wenn sie mit solchen ungesättigten Substanzen sich additionell vereinigen, welche die Gruppe<smiles>C=C(C)C</smiles>

enthalten, zu Verbindungen führen, die den elektro-negativen Theil des addirten Moleküls an das tertiär gebundene Kohlenstoffatom gebunden besitzen.

\section{Oxydation der I'iglinaldehydsulfonsäure.}

Einen weiteren Beweis für die bereits gegebene Deutung der Reaction zwischen schwefliger Sänre und Tiglinaldehyd darf man in der leichten Überführbarkeit der dabei entstehenden Mono- und Disulfonsäure in sulfonirte Valeriansäure erblicken unter dem Einflusse von Brom und Wasser, eines bekanntlich mild wirkenden Oxydationsmittels.

$\mathrm{Zn}$ einer aus $12 \mathrm{~g}$ Aldehyd in bereits beschriebener Weise dargestellten Lösung des Baryumsalzes der Mono- und Disulfonsäure wurde so viel Brom hinzngefügt, dass dieselbe auch nach 
mehrtägigem Stehen im Sonnenlichte eine starke Bromfarbe bei bebielt. Während dieser Operation schied sich Baryumsulfat aus, dessen Menge $22 g$ betrig. Wäre aus der angewandten Menge Tiglinaldehyd bloss oxypentandisulfonsaures Baryum in theoretischer Menge entstanden, so hätten $33.28 \mathrm{~g}$ Baryumsulfat gefunden werden müssen gemäss der Gleichung;

$$
\begin{aligned}
\mathrm{C}_{5} \mathrm{H}_{10} \mathrm{O}\left(\mathrm{SO}_{3}\right)_{2} \mathrm{Ba}+2 \mathrm{Br}_{2} & +2 \mathrm{H}_{2} \mathrm{O}=\mathrm{C}_{5} \mathrm{H}_{10} \mathrm{O}_{2} \mathrm{SO}_{3}+\mathrm{BaSO}_{4}+ \\
& +4 \mathrm{HBr} .
\end{aligned}
$$

Die Lösung wäre in diesem Falle baryumfrei gewesen.

Wäre aber der Tiglinaldehyd durch die Einwirkung der schwefligen Säure voliständig bloss in valeraldehydsulfonsaures Baryum übergeführt worden, so hätte die Oxydation nach der Gleichung:

$$
\left(\mathrm{C}_{5} \mathrm{H}_{9} \mathrm{OSO}_{3}\right)_{2} \mathrm{Ba}+2 \mathrm{Br}_{2}+2 \mathrm{H}_{2} \mathrm{O}=\left(\mathrm{C}_{5} \mathrm{H}_{9} \mathrm{O}_{2} \mathrm{SO}_{3}\right)_{2} \mathrm{Ba}+4 \mathrm{HBr}
$$

verlaufen miissen, welche die Bildung von Baryumsulfat ansschliesst und fordert, dass die Lösung auch nach der Oxydation noch baryumhältig sei. In Wirklichkeit war im Filtrat vom Baryumsulfat noch Baryum nachweisbar. Da, wie es sich weiter zeigen wird, die Bildung der sulfonirten Valeriansäure nachgewiesen werden konnte, so ist auch durch diese Beobachtung. festgestellt, dass durch die Addition von schwefliger Säure zu Tiglinaldehyd sowohl Monosulfonsäure als Disulfonsäure gebildet wird.

Nach vollendeter Oxydation wurde der Überschuss des Broms durch Erhitzen und gleicbzeitiges Durchleiten eines Gasstroms beseitigt, rom Baryumsulfat abfiltrirt und das Filtrat durch genaues Ausfällen mit Schwefelsänre vom Baryum befreit; hierauf successive mit Bleioxyd, Schwefelwasserstoff, Silberoxyd und abermals Schwefelwasserstoff die vorhandene Bromwasserstoffsäure entfernt und schliesslich concentrirt. Die concentrirte Lösung wurde in zwei Theile getheilt und aus einem durch Kochen mit Silberoxyd das Silbersalz, aus dem andern durch Versetzen mit Baryumearbonat das Baryumsalz dargestellt.

Das Silbersalz krystallisirt in farblosen gestreiften Prismen, die sich beim langsamen Auskrystallisiren zu kleinen Büscheln zu sammensetzen. Das Salz scheint mässior zersetzlich, löst sich 
schwer in kaltem Wasser, leichter in heissem, und enthält kein Krystallwasser.

Die Silberbestimmung wurde durch Titration nach Volhard ausgeführt.

$0.3029 \mathrm{~g}$ Substanz entsprachen $17.9 \mathrm{~cm}^{3}$ einer Rhodankalium-

lösung, von der $1 \mathrm{~cm}^{3}=0.003008 \mathrm{~g}$ Cl. $0 \cdot 2475 g$ Substanz gaben $0 \cdot 1365 g \mathrm{CO}_{2}$ und $0 \cdot 047 g \mathrm{H}_{2} \mathrm{O}$.

Berechnet für

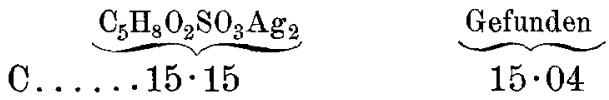

$$
\begin{aligned}
& \mathrm{H} \ldots 2 \cdot 11 \quad 2 \cdot 02 \\
& \mathrm{Ag} \ldots .54 \cdot 28 \quad 54 \cdot 54
\end{aligned}
$$

Das Baryumsalz krystallisirt in farblosen rhombischen Tafeln. Es ist in kaltem Wasser ziemlich leicht löslich, in heissem nicht merklich leichter. Es enthält ein Molekül Krystallwasser, welches es erst bei $150^{\circ}$ vollständig verliert.

$0.1762 g$ Substanz gaben $0.0475 g \mathrm{H}_{2} \mathrm{O}$ und $0.115 \mathrm{~g} \mathrm{Co}_{2}$. $0.393 g$ Substanz gaben $0.2725 \mathrm{~g} \mathrm{BaSO}_{4}$.

$0.255 g$ Substanz verloren beim Erhitzen auf $150^{\circ}$ bis zur Gewichtsconstanz $0 \cdot 014 \mathrm{~g} \mathrm{H}_{2} \mathrm{O}$. Bei höherem Erhitzen (auf $\left.175^{\circ}\right)$ blieb das Gewicht unverändert.

\begin{tabular}{|c|c|}
\hline $\mathrm{C}_{5} \mathrm{H}_{8} \mathrm{O}_{2} \mathrm{SO}_{3} \mathrm{Ba}+\mathrm{H}_{2} \mathrm{O}$ & Gefunden \\
\hline C....17.91 & $17 \cdot 80$ \\
\hline Н ... 2.98 & $2 \cdot 99$ \\
\hline Ва....40.89 & $40 \cdot 77$ \\
\hline $\mathrm{H}_{2} \mathrm{O} \ldots 5 \cdot 37$ & $5 \cdot 49$ \\
\hline
\end{tabular}

Berechnet für

Zum Schlusse sei es mir gegönnt, meinem hochverehrten Lehrer, Herrn Professor Lieben, sowie Herrn Dr. Zeisel für die berathende Unterstiatzung, die sie mir während des Verlaufs der Arbeit in freundlichster Weise angedeihen liessen, meinen innigsten Dank auszusprechen. 